\title{
Le management de continuité d'activité en période de crise sanitaire : cas des entreprises de FES Shore
}

\section{Business continuity management during a health crisis: the case of FES Shore companies}

\author{
Mme. HABBANI Souad \\ Enseignant chercheur \\ Faculté des sciences juridiques, économiques et sociales, Fès \\ Laboratoire Etudes et recherche en Management des organisations et des territoires (ERMPT) \\ Mlle. EL JAMOUSSI Yasmina \\ Doctorante \\ Faculté des sciences juridiques, économiques et sociales, Fès \\ Laboratoire Etudes et recherche en Management des organisations et des territoires (ERMOT)
}

\begin{abstract}
Résumé : Le Maroc, à l'instar du monde souffre de la propagation du coronavirus, cette pandémie qui a mis en danger non seulement l'existence de l'être humain, mais a impacté également l'économie nationale et internationale, cette dernière a été estimé en tant que pire des crises qu'à connu le monde depuis la guerre mondiale, ce qui amène a posé la question sur la place des entreprises en tant que premières entités impactées par cette crise et leurs comportement afin d'assurer la continuité d'activité et de minimiser le maximum possible les conséquences négative sur la rentabilité.

Le management de continuité d'activité intervient donc pour aider les entreprises à faire face à des situations imprévues comme le cas du covid 19, pouvant affecter son fonctionnement normal.
\end{abstract}

Mots clés : Crise, risque, offshore, management de continuité d'activité, coronavirus.

\begin{abstract}
Morocco, like the rest of the world, is suffering from the spread of the corona virus, a pandemic that has not only endangered the existence of human beings, but has also impacted the national and international economy, which has been estimated as the worst crisis that the world has known since the World War, which leads to the question of the place of companies as the first entities impacted by this crisis and their behavior in order to ensure the continuity of activity and to minimize as much as possible the negative consequences on the profitability.

Business continuity management therefore comes into play to help companies deal with unforeseen situations such as the case of covid 19 , which may affect its normal operation.
\end{abstract}

Keywords: Crisis, risk, offshore, business continuity management, corona virus.

Digital Object Identifier (DOI): https://doi.org/10.52502/ijesm.v1i1.161

This work is licensed under a Creative Commons Attribution-NonCommercial-NoDerivatives 4.0 


\section{Introduction}

C'est dans les contextes les plus difficiles ou naissant les opportunités de changement pour les organisations. Par les temps qui courent, les entreprises aujourd'hui doivent faire preuve de résilience et de forte adaptabilité à la situation actuelle pour assurer leur survie.

Les menaces d'aujourd'hui sont sans cesse plus nombreuses et les entreprises sont confrontées à un environnement de plus en plus complexe et instable cherchent à formaliser une politique d'anticipation des risques et de gestion de leurs conséquences en cas de crise, d'où l'importance de mettre en place un système de gestion de crise permettant de maitriser l'activité durant toutes les étapes de la crise.

La propagation de la pandémie grippale du coronavirus, a fait apparaître un ensemble de dysfonctionnement en matière de management des situations difficiles, et chaque lacune même secondaire peut devenir un facteur de risque, la crise a permis de révéler des faiblesses peu visibles en période de stabilité.

Le secteur d'offshoring faisant partie des secteurs à forte valeur ajoutée qui contribue à la création des emplois et constitue un moteur de développement pour le Maroc, a été impacté par la survenue de cette crise inédite, ce qui a nécessité la mise en place en urgence d'un plan d'action afin d'assurer la continuité d'activité en mode dégradé pour les projets opérants dans les secteurs vitaux, et cela pour compenser les pertes engendrer par l'arrêt total des activités liées aux branches de tourisme, transport aérien, immobilier et automobile.

L'objectif de notre étude c'est de mettre en avant l'importance de la mise en place d'un système de gestion bien structuré en cette période, permettant d'assurer la continuité d'activité face à une concurrence rigide qui adopte des outils efficaces pour la gestion des crises et de minimiser le maximum possible l'impact négatif de celle-ci sur la continuité d'activité.

Donc, Suite à la propagation de la pandémie du COVID 19 qui a paralysé l'activité économique au Maroc, nous avons arrêté la problématique de notre recherche sous forme des questions suivantes :

- Comment les entreprises offshores ont pu faire face à cette crise ?

- Quelle était l'efficacité des mesures prises afin d'assurer la continuité d'activité durant cette période?

- Quelles sont les leçons tirées de cette expérience?

\section{Cadre théorique}

\section{Le concept du management de continuité d'activité et son cadre législatif}

Cette partie vise à délimiter le champ conceptuel du management de continuité d'activité, et de présenter une vue de synthèse sur le cadre réglementaire.

\subsection{Le management de continuité d'activité : un concept à deux approches}

La définition du management de continuité d'activité varie selon quel ongle nous traitons celui-ci, à travers la littérature ce concept revêt deux approches, celle préventive dite optimiste, et celle par conséquence dite pessimiste.

\section{- Approche préventive}

Dans cette optique les définitions du management de continuité d'activité reposent sur l'anticipation et la préparation des risques afin de réduire leur impact et garantir la continuité pour les activités critiques. 
Le management de continuité d'activité a été défini par la BCI (Business Continuity Institute) comme étant « un processus holistique de management qui identifie les impacts potentiels qui menacent une organisation et fournit un cadre pour assurer la résilience de l'entreprise et construire une réponse efficace qui protège les intérêts, la réputation et les activités de création de valeurs de l'organisation » ( Bennasar, 2010), cette définition met en avant ce type de management qui permet de détecter les risques et les crises qui peuvent confronter l'organisation afin d'assurer sa résilience sans mettre en danger son existence, de cela en peut déduire que le management de continuité d'activité est devenue une partie intégrante du management classique qui intervient dès l'apparition d'un sinistre ou bien d'une crise, l'introduction de cette nouvelle fonction consiste à remplacer le système traditionnel par un autre plus profond et organisé et variant en fonction du contexte de l'entité.

En déduit de la définition, que le management de continuité d'activité est un processus guidé qui permet d'aboutir à un résultat bien déterminé.

La deuxième définition de la CRBF (Le comité de la réglementation Bancaire et Financière) le définit comme un « ensemble de mesures visant à assurer, selon divers scénarios de crise, y compris face à des chocs extrêmes, le maintien, le cas échéant de façon temporaire selon un mode dégradé, des prestations de services essentielles de l'entreprise puis la reprise planifiée des activités » (Bennasar, 2010).

Celle-ci met en avant d'une part la notion de temps qui est primordiale afin de déterminer la période d'interruption des activités et le temps de reprise, et d'autre part l'importance du secteur d'activité qui détermine les services essentiels sur lesquelles repose l'activité (Coursaget, 2015).

\section{- Approche conséquentialiste}

$\mathrm{Au}$ niveau de cette approche, en considère l'impossibilité de prévenir tous les risques et les crises qui peuvent survenir, ce qui nécessite de la part de l'entreprise, une capacité à absorber les chocs externes face à l'évolution et la multitude des risques ainsi que leur caractère complexe et rapide.

Et donc le management de continuité d'activité est défini comme une «approche globale qui comprend la politique, les règles et les procédures pour garantir le maintien ou la reprise des opérations spécifiées d'une façon planifiées en cas de perturbation. Son but est de réduire au minimum les conséquences opérationnelles, financières, légales, de réputation et autres conséquences substantielles résultant d'une perturbation » (Ruelle, 2012).

La mise en place du management de continuité d'activité se traduit par la mise en place du plan de continuité d'activité qui l'opérationnalise sous forme de procédures et techniques.

Le plan de continuité d'activité est considéré comme un ensemble de procédures et dispositions prévues pour permettre à l'entreprise de réagir à un sinistre, de manière à garantir la reprise de ses activités critiques (Ruelle, 2012).

Un plan de continuité d'activité a pour objet de décliner la stratégie et l'ensemble des dispositions qui sont prévues par une organisation pour garantir la reprise et la continuité de ses activités à la suite d'un sinistre ou d'un événement perturbant gravement son fonctionnement normal (Coursaget Haas, 2015). 


\subsection{Cadre réglementaire du management de continuité d'activité en cas de crise de pandémie grippale}

Le cadre réglementaire international affiche une multitude de lois qui donne au management de continuité un caractère obligatoire, ce qui impose à l'entreprise de détenir un plan de continuité d'activité ( les accords de Bâle, le CRBF 2004, la loi sarbane oxley) ou bien un caractère facultatif mais qui permettent de conférer à l'organisation un label en matière de gestion des risques et des crises.

En matière de gestion des pandémies, la loi française a travers le plan de prévention et de lutte « pandémie grippale » $\mathrm{n}^{\circ}$ 850/SGDSN/PSE/PSN octobre 2011, mis l'accent sur le dispositif à mettre en place et les mesures stratégiques à entreprendre en cas de pandémie grippale, qui en outre de son impact sanitaire, créent des perturbations dans la vie économique et sociale (le plan de prévention et de lutte «pandémie grippale », 2011).

$\mathrm{Au}$ Maroc, il y a une forte concentration des lois sur le secteur financier ( directive relative au plan de continuité de l'activité au sein des établissements de crédit, circulaire $n^{\circ} 01 / 10 \mathrm{du}$ conseil déontologique des valeurs mobilières, circulaire $n^{\circ}$ DAPS/EA/08/11 relative au contrôle des entreprises d'assurance et de réassurance), vu le caractère obligatoire qu'ils relèvent.

En revanche, pour les autres secteurs d'activité la loi n'exige aucune tenue des procédures en matière de gestion de risques et de crises, et il y a une absence complète de recommandation en matière de gestion des pandémies grippale, suite à cette insuffisance les entreprises aujourd'hui qui sont confrontées à la crise du Coronavirus se trouvent en face d'une crise sans précédente et sans disposer des outils nécessaires pour faire preuve de résilience ou même développée des outils vus la fréquence avec laquelle cette pandémie s'est étalé.

\section{Management de continuité d'activité : Apport et démarche}

Le management de continuité d'activité fournit un plan de continuité d'activité pour affronter diverse perturbations, une démarche qui vise à accroitre la résilience des organisations, à maintenir ou rétablir le fonctionnement à un niveau acceptable, tout en minimisant les impacts des défaillances qui pourraient survenir.

\subsection{Apport du management de continuité d'activité en situation de crise}

Dès qu'on parle du management de continuité d'activité cela renvoie à une situation qui va générer l'arrêt total ou partiel du cycle d'exploitation considéré comme inattendu et imprévisible est ce caractère est spécifique aux risques et aux crises, ce qui fait que ces deux dès leurs apparitions déclenchent un processus pour les gérer et les maitriser tout en faisant recours à un plan de continuité d'activité.

Le plan de continuité d'activité est composé donc de trois parties, chacune correspondant à une étape précise du déroulement d'une crise, la gestion d'une crise s'inscrit dans une temporalité bien définie (Cavallari, Hassid, 2011). 


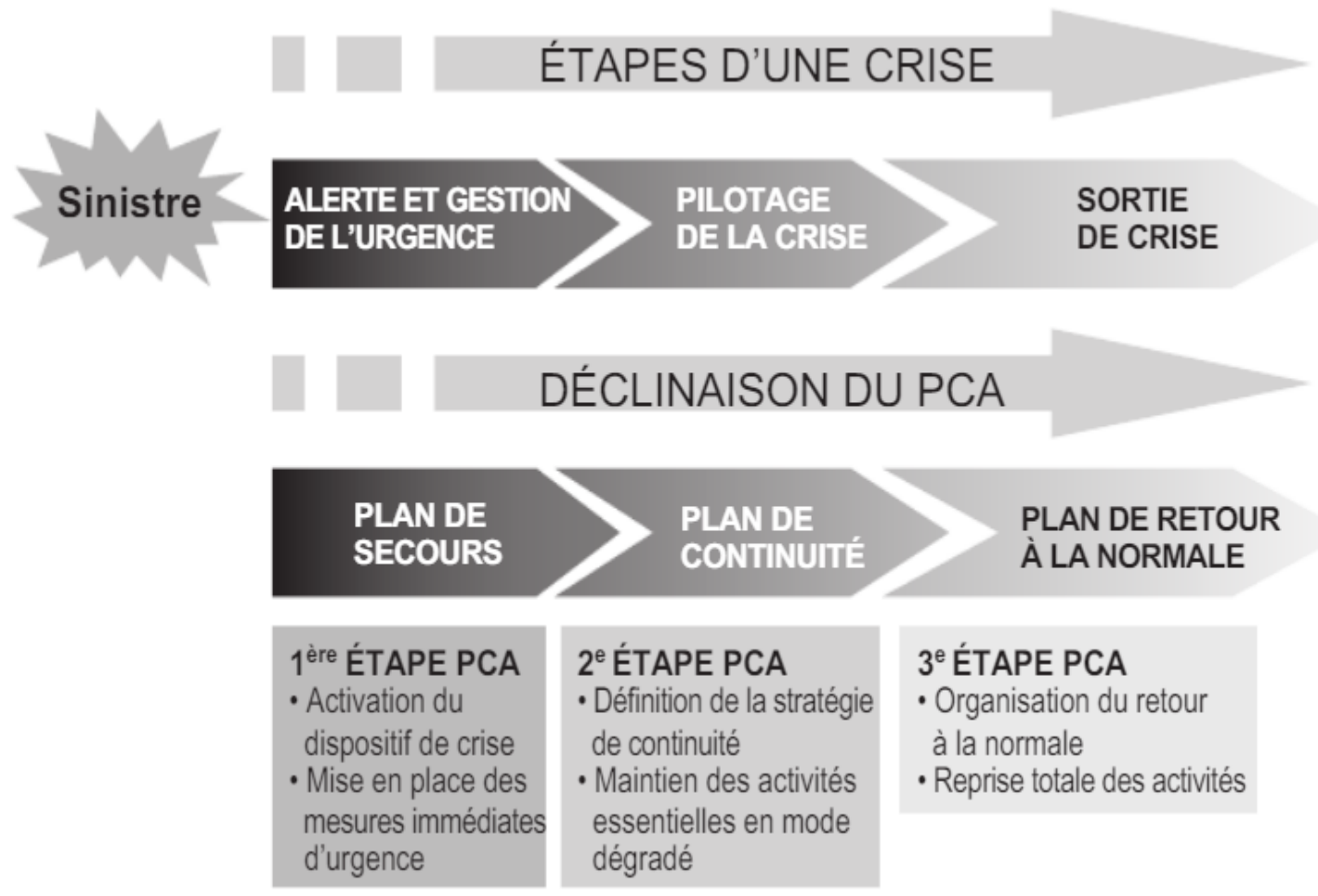

Source : O.CAVALLARI ET O. HASSID., Réaliser le plan de continuité d'activité de son entreprise : Guide opérationnel., 2011.

Le PCA a pour objectif de mettre en place un dispositif pour (Cavallari Hassid, 2011) :

- Organiser la réaction opérationnelle des collaborateurs en cas de survenance d'une crise ;

- Maintenir les activités essentielles en mode dégradé lors d'une crise ;

- Organiser la sortie de crise pour un retour à la normale des activités de l'entreprise.

\subsection{La démarche du management de continuité d'activité}

Dès que l'activité de l'entreprise est compromise suite à la survenue d'une situation de crise, un dispositif de management spécifique à la situation doit être mise en place afin de minimiser les conséquences de cet événement sur l'activité.

De ce fait, il est nécessaire d'identifier les activités critiques de l'entreprise et les ressources critiques pour les maintenir, cette tâche non aisée, demande l'implication des dirigeants afin de garder les activités qui assurent la pérennité, et cela avec prise en compte des impacts potentiels et des pertes induites pour l'organisation.

Après l'identification des activités critiques, il faut attribuer à chacune d'entre elles les ressources critiques, tout en réduisant l'indépendance aux ressources indispensables par le moyen des ressources de substitution.

Une fois les activités et les ressources essentielles sont fixées, vient l'étape de définition des scénarios de crise qui les impactent, et cela se fait par le moyen du Business Impact Analysis (BIA), «qui consiste à étudier comment les sinistres, lorsqu'ils se produisent, affectent le déroulement des activités critiques de l'entreprise »(Besluau, Guinet, 2010), et cela a pour objectif d'élaborer une stratégie de continuité d'activité pendant la période de crise, qui consiste en «l'identification des effets à produire et à coordonner dans l'espace et dans le temps afin de maintenir l'activité de l'organisation à un niveau prédéfini malgré l'occurrence des scénarios de risques qui ont été retenus » (Coursaget A, Haas L, 2015). 


\subsection{Le facteur humain : clés du maintien de la continuité d'activité}

Le facteur ressource humain joue un rôle primordial dans le management de continuité d'activité de telle sorte que ces personnes agiront au sein de l'entreprise afin de garantir le suivi de l'activité sans aucune interruption. En revanche, en cas de crise, le personnel est confronté à un état de stress et d'anxiété qui impacte négativement sa productivité ce qui nécessite de la part de l'organisation une gestion très prudente durant cette période.

Le stress et l'anxiété affectent automatiquement le rendement de l'être humain surtout en cas de crise pandémique, ce qui conduit à une détérioration de la qualité du travail due à une multiplicité des erreurs commises, manque de motivation et de créativité.

Le management de continuité d'activité doit prendre en considération la gestion du stress organisationnel afin de se préparer à l'avance et savoir réagir rapidement afin d'éviter la perte de compétences jugées clés pour le bon fonctionnement des services de l'entreprise, et cela demande de sa part un investissement énorme vue les coûts chères du coaching et de l'accompagnement psychologique, mais qui reste incomparable au coût financier que génère le stress à l'entreprise tel que les coûts liés à la perte de compétence, formations du personnel, absentéisme.

De plus les coûts réels du stress sont vraisemblablement nettement supérieurs car il faut également prendre en compte les coûts indirects du stress, tels que les pertes de productivité, l'altération du rapport au client et les problématiques interpersonnelles que les situations de stress peuvent engendrer. Cela impose à l'organisation de maintenir le moral des équipes en situations difficiles (garder le calme, montrer l'optimisme et rester à l'écoute) et d'augmenter l'énergie des collaborateurs (Berghmans,2010).

\section{Cadre pratique : Analyse de l'efficacité du management de continuité d'activité au sein des entreprises offshore en période de crise du coronavirus.}

Le contexte actuel revêt une concurrence intense qui oblige les entreprises de développer leur stratégie de management surtout en période de crise, et faire preuve de résilience, dans l'objectif non seulement de garantir la pérennité, mais de renforcer également sa compétitivité et gagner la confiance des différentes parties prenantes.

Le but de cette partie c'est de confronter les connaissances que nous avons rassemblées dans la phase théorique avec la réalité du terrain.

\section{Méthodologie et choix de l'échantillon}

Dans ce paragraphe nous présenterons la principale méthodologie suivie pour mener à bien notre étude, ainsi que la structure de l'échantillon choisit.

\subsection{Méthodologie de l'étude}

Au niveau de cette étude, nous avons choisi de passer par une méthode qualitative basée sur l'observation et les entretiens visant à étudier les stratégies mises en place et le management effectué en situation de crise et sa capacité à assurer la continuité d'activité.

Les entretiens ont été réalisés de manière individuelle et semi-directive, La durée des entretiens a pris entre trente minutes et une heure. En revanche, il est à noter qu'au niveau de cette étude qualitative, aucun logiciel de traitement de données n'a été utilisé, nous avons uniquement regroupé les idées qui se rejoignent dans le sens de la thématique. 


\subsection{Choix de l'échantillon}

Notre choix d'échantillon s'est orienté vers les entreprises qui opèrent dans le secteur offshore, qui constitue aujourd'hui un véritable moteur de croissance pour l'économie marocaine, en raison de son potentiel de création d'emplois et de sa contribution à la balance commerciale du pays.

Ce secteur a connu un fort ralentissement suite à la propagation de la pandémie du coronavirus, l'impact de cette dernière était en fonction du secteur d'activité dans lequel opère le client donneur d'ordre.

Le choix de l'échantillon repose sur deux critères primordiaux à notre étude :

Le premier critère c'est l'existence d'un réel dispositif de management en temps de crise afin d'assurer la continuité d'activité durant cette période ;

Et le deuxième, c'est le risque élevé dont lequel est confronté les entreprises offshore, vu la délocalisation de l'activité, qui rend le suivi de la bonne application des procédures difficiles a gérer à distance.

\begin{tabular}{|l|l|l|}
\hline \multicolumn{1}{|c|}{ Fonction de l'Interviewés } & \multicolumn{1}{|c|}{ Entreprises } & \multicolumn{1}{c|}{ Domaine d'activité } \\
\hline Chef de projet & Sitel Group & Business Process Outsourcing \\
\hline Directeur informatique & CGI & $\begin{array}{l}\text { Information } \\
\text { Outsourcing }\end{array}$ \\
\hline $\begin{array}{l}\text { Directeur des ressources } \\
\text { humaines }\end{array}$ & Alten & $\begin{array}{l}\text { Information } \\
\text { Outsourcing }\end{array}$ \\
\hline
\end{tabular}

\section{Présentation et analyse des résultats}

Afin de répondre à nos questions de recherche, dans cette partie nous visons à présenter et analyser l'ensemble des résultats obtenus des entretiens réalisés auprès des entreprises de Fès shore.

\subsection{Présentation des résultats de l'étude}

- Management de risque au sein des sociétés offshore : organisation et réglementation

Selon l'entretien effectué avec l'ensemble des sociétés de Fès shore, les répondants ont confirmé qu'il n'existe pas un service bien précis dédiés au management des risques et crises, cependant suite à la crise du covid 19 , les responsables ont réunis les directions des entreprises (direction générale, direction des ressources humaines, direction de communication, direction des opérations, direction de compte, direction financière...), afin de gérer la situation.

Le responsable d'Alten a confirmé que «la société dispose d'un CODIR qui se réunit à chaque fois qu'il y a des imprévus pour gérer et trouver des solutions à la situation présenté ».

\begin{tabular}{|l|l|l|l|}
\hline Société & $\begin{array}{l}\text { Direction de management de } \\
\text { crise }\end{array}$ & $\begin{array}{l}\text { Existence d'un référentiel } \\
\text { législatif }\end{array}$ & Certification \\
\hline Sitel Group & Comité de direction & Oui, obligatoire & $\begin{array}{l}\text { Label de conformité } \\
\text { sanitaire }\end{array}$ \\
\hline CGI & Business Unit & Oui, obligatoire & ISO 27001 \\
\hline Alten & CODIR & Non, facultatif & aucun \\
\hline
\end{tabular}


D'après le tableau qui résume la situation des entreprises offshore, on déduit que l'entreprise Sitel est la seule qui dispose d'une certification de conformité sanitaire délivré par l'AFNOR permettant de valoriser la conformité aux mesures sanitaires visant la protection des collaborateurs pendant la période du covid 19.

Quant à CGI, il dispose d'une certification ISO liée essentiellement à son activité principale alors qu'Alten ne dispose d'aucune certification, cela s'explique par le fait que ces deux sociétés avaient la possibilité d'assurer l'activité entière en distancielle, contrairement à Sitel Group qui disposait de certain poste nécessitant la présence obligatoire sur site.

Le Label obtenu par Sitel repose sur une charte de prévention complète mise en place par l'AMMC, et qui prend en compte les exigences de l'OMS, les bonnes pratiques d'hygiène sur les lieux de travail ainsi que les recommandations internationales en matière de protection de la santé au travail.

Sitel a été considéré comme le premier acteur de l'AMRC à avoir lancé les audits sur ces différents sites, et à travers lequel Sitel s'engage à

- Garantir la continuité d'activité tout en respectant les exigences de la charte de conformité sanitaire de l'AMRC ;

- Afficher une totale transparence en fournissant des preuves et un contrôle externe indépendant pour les collaborateurs, les donneurs d'ordre et les autorités nationales ;

- Gagner en crédibilité en annonçant ses pratiques et leur niveau de conformité ;

- Standardiser ses pratiques en matière de prévention et de lutte contre le COVID 19.

\section{- Impacts du COVID 19 sur les entreprises offshores}

La crise avait des impacts négatifs et positifs sur les sociétés offshores et cela en fonction de la branche d'activité qu'elles gèrent :

\begin{tabular}{|l|l|}
\hline Société & Impact de la crise du covid 19 \\
\hline Sitel Group & Négatif \\
\hline CGI & Aucun impact \\
\hline Alten & Négatif et positif \\
\hline
\end{tabular}

Les répercussions de la crise du covid 19 a varié d'une entreprise à une autre et cela en fonction de l'activité exercée, selon les répondants :

Pour Alten, la crise avait un impact positif sur l'activité des réseaux et télécommunications où l'entreprise a gagné de nouveau projet suite à une forte augmentation de la demande dans ce domaine, cependant, pour l'industrie automobile l'activité à connu une forte baisse accompagné de licenciement des salariés.

Pour CGI la crise n'avait aucun impact vu que l'activité informatique pouvait être assurée à distance et le projet n'a connu aucun impact considérable.

Pour Sitel Group, la crise avait un impact négatif sur l'ensemble des projets de l'entreprise avec une baisse du flux d'activité et la baisse du chiffre d'affaire, auxquelles s'ajoute :

- L'augmentation du taux du turn over ;

- L'augmentation de l'absentéisme ;

- Baisse de la performance des collaborateurs ;

- Augmentation de certaines charges. 
Ces entreprises ont bien confirmé qu'ils n'ont jamais rencontré une crise pareille et ils l'ont jugé inédite en termes de gestion, et cette crise n'a jamais été prévue parmi les scénarios de risques dont elles ont l'habitude de gérer.

Cependant, les répondants ont bien confirmé qu'ils disposent d'un plan de continuité d'activité qui permet d'identifier et de formaliser les solutions de contournement des sinistres majeurs pouvant mettre fin à la continuité d'activité.

De plus, ces entreprises ont utilisés durant cette période divers outils de gestion de crise :

- Création d'une cellule de crise ayant pour objectif la gestion et la prise de décisions nécessaire à la bonne marche d'activité ;

- La communication de crise interne et externe pour informer les collaborateurs et parties prenantes.

- Mesures de continuité d'activité en période de crise

Afin d'assurer la continuité du cycle d'exploitation, les entreprises ont pris des mesures sur plusieurs niveau :

- Mesures sur le plan sanitaire :

Sensibilisation des salariés ;

Désinfection quotidienne des plateaux ;

Distribution des gels nettoyants et des bavettes ;

Interdiction de déplacement sur site qu'après accord de la hiérarchie.

- Mesures sur le plan de gestion des ressources humaines :

Cotisation pour les salariées ayant leur salaire impacté par la crise ;

Réduction des horaires de travail ;

Arrêt temporaire du travail pour les salariées le souhaitant ;

Accord des indemnités de la CNSS pour arrêt de travail ;

Accord des autorisations de déplacement en période de quarantaine et couvre-feu ;

Gratuité du transport pour le compte des salariés ;

Application du télétravail soit à cent pourcent ou en combinaison du présentiel et à distance ;

Validation des congés sans solde ;

Suspension de contrat de travail ;

Accompagnement des cas contaminé par le virus.

\section{- Mesures sur le plan administratif}

Fermeture des parties communes (cafeteria, salle de jeux, salle de prière) ;

Désactivation des badges et portes automatiques ;

Réduction des groupes pour la partie formation initiale de 14 à 6 personnes et arrêt des formations continues ;

Suspension du papier et dématérialisation des démarches administratifs ;

\section{- Répercussion des mesures prises sur la continuité d'activité}


L'arrivé de cette crise inédite a obligé les entreprises à prendre des mesures afin de garantir la continuité d'activité, selon les répondants les entreprises ont pu assurer soit totalement ou partiellement les activités, sans aucun arrêt totale qu'il soit temporaire ou définitif.

\begin{tabular}{|l|l|}
\hline Société & Déroulement de l'activité \\
\hline Sitel Group & Assurer partiellement \\
\hline CGI & Assurer totalement \\
\hline Alten & Assurer partiellement \\
\hline
\end{tabular}

Selon le tableau ci-dessus, la société CGI a réussi à maintenir son activité en totalité grâce au recours au télétravail contrairement à Alten et Sitel Group qui en raison de la spécificité de certaines activités, ceux-ci n'ont été assuré que partiellement est cela est dû soit à la baisse du flux d'activité ou la suspension temporaire de certain projet surtout ceux liées à la vente.

Afin de compenser les pertes générées par les activités en arrêt, les entreprises ont bénéficié de l'aide de l'Etat, ainsi qu'elles ont fait recours à la mobilité des collaborateurs sur les différentes activités.

\section{- Apprentissage et retour d'expériences}

Selon les répondants, cette période a révélé plusieurs difficultés en terme de gestion en situation de crise :

- Difficulté de prise de décision ;

- Gestion de la panique en cas de détection d'un cas contaminé par le virus ;

- Difficulté de communication en l'existence de rumeur entre les collaborateurs et les fausses informations ;

- Augmentation du niveau des incidents techniques surtout avec la mise en place du télétravail ;

Il est vrai que cette crise avait un impact négatif sur les entreprises offshores avec toutes les difficultés de gestion qu'accompagnait cette période. Or, la crise est considérée également comme une opportunité d'apprentissage et de préparation de nouveaux défis auxquelles l'entreprise peut être confrontée, les répondants ont bien expliqué que grâce à cette crise plusieurs changements peuvent être appliqués dans le futur :

- L'ajout de l'ensemble des problèmes rencontrés et les solutions trouvées au plan de continuité d'activité ;

- L'option pour le télétravail en tant que moyen de réduction des charges.

\subsection{Analyse et discussion des résultats}

L'analyse des entretiens a permis d'approfondir et de confirmer certains constats que nous avons déjà débattus dans l'examen de la littérature mais également d'enrichir les écrits sur les démarches adoptées par une catégorie particulière d'entreprises, celles du secteur offshore qui sont considérées comme de fortes entreprises en plein développement et soumises à des procédures et réglementations internationales.

En effet, les résultats obtenus lors de l'étude qualitative ont pu confirmer que durant cette pandémie c'est l'approche conséquentialiste du management de continuité qui a été appliquée vu que toutes les entreprises n'ont pas pu prévenir une crise pareil et donc devrait faire appel à leurs capacités résilientes afin de garantir la continuité d'exploitation. 
En ce qui concerne le référentiel réglementaires, ces entreprises ne sont pas y soumis obligatoirement du point de vu juridictionnel, mais l'objectif phare pour certaine c'est de se labéliser afin de gagner la confiance des parties prenantes et répondre à la demande des clients donneur d'ordre.

Comme toute entreprise au monde, le secteur offshore a eu des impacts considérables sur l'activité globale, et les sociétés de Fès shore ont subi des pertes que ce soit sur le plan financier ou humain, malgré les efforts engagés et les mesures prises pour mettre sous contrôle la situation.

Certes, selon les répondants, la crise a introduit des changements qui non jamais été accepté par la hiérarchie et qui ont été jugé efficace et rentable permettant de réduire les charges, d'accélérer le traitement et d'augmenter la performance organisationnelle.

\section{Conclusion}

Le management de continuité d'activité est une branche de la gestion des risques qui permet de garantir la pérennité de l'entreprise suite au déclenchent d'un événement majeur. Aujourd'hui les organismes sont conscients de l'importance d'adopter une gestion efficace des crises.

Les sociétés offshores enquêtées, même s'elles ne disposent pas d'un service spécifique à la gestion des risques et crises, elles possèdent les outils nécessaires pour faire face à de telles situations.

En réponse aux questions de recherche déjà posées, on déduit que l'ensemble des mesures et décisions prises ont été jugée efficaces vu que pour certaine ils ont garanti la marche normale de l'activité et pour d'autre celle-ci a été maintenue partiellement sans avoir un arrêt définitive mettant en danger l'existence de l'entité.

L'arrivée de la crise du covid 19 a dévoilé plusieurs dysfonctionnements au niveau de la gestion des périodes difficiles. En effet, les entreprises avaient des contraintes d'ordre social technique et financière et qui avaient du mal à gérer.

En revanche, cette situation était une source d'apprentissage pour les gestionnaires et un facteur de changement radical introduisant de nouvelles pratiques au sein des entreprises.

\section{Bibliographie}

[1] A.COURSAGET, L.HAAS. Le plan de continuité d'activité (PCA) : Approche méthodologique. Sécurité et stratégie. 2015 ;

[2] A.TAMMINEEDI R. (2010), Business Continuity Management: A Standards-Base Approach, Information Security Journal: A Global Perspective ;

[3] C. BERGHMANS. Stress au travail : Des nouveaux outils pour les ressources humaines. Dunod; 2010 ;

[4] Circulaire du Le plan de prévention et de lutte «pandémie grippale » $n^{\circ}$ 850/SGDSN/PSE/PSN octobre 2011 ;

[5] Circulaire $\mathrm{n}^{\circ} 1 / 10$ du conseil Déontologique des Valeurs Mobilières ;

[6] Comité de Bâle sur le contrôle bancaire (2004) ;

[7] E.BESLUAU, D GUINET. Management de la continuité d'activité assurer la pérennité de l'entreprise: planification, choix techniques et mise en œuvre. Bagneux: Numilog; 2010 ;

[8] O.CAVALLARI, O HASSID. Réaliser le plan de continuité d'activité de son entreprise : Guide opérationnel. Maxima; 2011 ;

[9] M. BENNASAR. Plan de continuité d'activité et système d'information: Vers l'entreprise résiliente Ed. 2. Dunod ; 2010

[10] S.RUELLE. Continuité d'activité et gestion de crise : de la technique à l'humain. Sécurité et stratégie. 2012.

[11] Z.ERRABIH. Management des risques et de la continuité d'activité : cas des grandes et moyennes entreprises de la région Rabat-casablanca, thèse doctaorale, Fès 2014. 